\title{
Zeigen, was man kann
}

_ Amsterdam. 20. Juni 2011, 19 Uhr. Der Weltkongress der Physiotherapeuten wird eröffnet. Die beiden ungarischen Artisten „La Vizio“ bezaubern mit ihrer Partner-Akrobatik die Gäste (॰ „WCPT-Kongress“, S. 12). Als nächster „Act“ schreitet Bas Eenhoorn zum Mikrofon, Vorsitzender des niederländischen Physiotherapieverbandes. Seine Worte lassen mich aufhorchen: „Kämpft für eine bessere Position innerhalb des Gesundheitssystems“, ermutigt er die Kongressteilnehmer. Das Ringen um Mitsprache auf politischer Ebene scheint also kein typisch deutsches, sondern ein weltweites

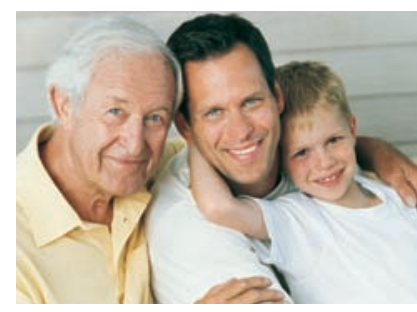
Problem zu sein. „Öffnet die Fenster, damit die Menschen sehen, was Physiotherapeuten leisten. Zeigt der ganzen Welt, was Physiotherapie kann“, ermuntert der Niederländer weiter.

_ Einige Länder nutzen die Olympischen Spiele, um Physiotherapie einer breiten Öffentlichkeit bekannt zu machen: Vertreter aus Australien (Sydney 2000), Kanada (Vancouver 2010) und Großbritannien (London 2012) sprachen in Amsterdam über ihre Erfahrungen. So wählte der kanadische Berufsverband im Vorfeld der Spiele physiotherapeutisch kompetente Interviewpartner und schulte diese im Umgang mit den Medien. Er wollte die Vielfalt der gesamten Physiotherapie in den Fokus rücken und nicht nur die Sportphysiotherapie. Die Medienkampagne zielte auf Frauen im Alter zwischen 35 und 45 Jahren, weil diese meist über die Gesundheit ihrer Kinder und Eltern entscheiden und somit wichtige Multiplikatoren sind. So war Physiotherapie in Kanada in aller Munde, und als Krönung der Kampagne dankte eine Goldmedaillengewinnerin vor laufender Kamera ihrem Physiotherapeuten.

_ Es muss nicht auf das nächste Sportgroßereignis gewartet werden, um den Menschen zu zeigen, was Physiotherapie kann. Öffentlichkeitsarbeit ist auch im Kleinen wirksam.

Herzlich, Ihre

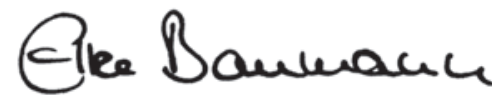

Angebote für jedes Lebensalter bietet die Physiotherapie. Doch viele Menschen wissen nichts davon.

\section{PHYSIOBONUS}

physiobonus heißen die Gewinnspiele in physiopraxis. Wer teilnehmen möchte, nutzt das Formular unter www.thieme.de/ physioonline in der Rubrik physioexklusiv. Und das gibt es in dieser Ausgabe zu gewinnen:

Bücher

3-mal „Physiotherapie am Kiefergelenk“"

Seite 36

3-mal „Zwischen Himmel und Erde"

Seite 47

3-mal „Die 100 besten

Cocktails“"

Seite 95

und außerdem

2-mal 2 Teleskopstöcke

Seite 53

2-mal „Zengarten \& Tee“

1 Erste-Hilfe-Koffer

Seite 58

Seite 61

1 Duschvorhang

„PrometheusWorld“

Seite 95

1 Umhängetasche „PrometheusWorld“ Seite 95 1 Familienzelt

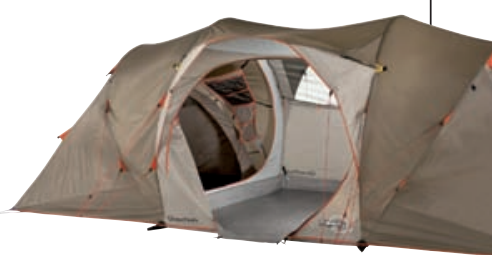

